\title{
Effects of Disorder in Two-Dimensional Quantum Antiferromagnets
}

\author{
C.M.S.Conceição AND E.C.MARINO \\ Instituto de Física \\ Universidade Federal do Rio de Janeiro \\ Cx.P. 68528 \\ Rio de Janeiro RJ 21941-972 \\ Brazil
}

\begin{abstract}
We study the effects of disorder in two-dimensional quantum antiferromagnets on a square lattice, within the nonlinear sigma model approach, by using of a random distribution of spin stiffnesses or zerotemperature-spin-gaps, respectively, in the renormalized classical and quantum disordered phases. The quenched staggered magnetic susceptibility at low temperatures is evaluated in each case. The large distance behavior of the quenched spin correlation function is also obtained in the quantum disordered phase. Disorder is shown to introduce a change from exponential to power-law decay in these functions, indicating that the spin excitations become gapless, in spite of the fact that the system is in a disorder state. A comparison is made with the dual behavior of skyrmion topological excitations in the renormalized classical phase.
\end{abstract}

PACS: 75.10.Jm, 75.10.Nr, 75.70.-i

Keywords: 2D antiferromagnets, disorder 


\section{1) Introduction}

The physics of two-dimensional quantum antiferromagnetic systems has been attracting a lot of interest, to a great extent due to the important role played by them in the high-Tc superconducting cuprates. The continuum description of these systems in terms of a Nonlinear Sigma Model (NLSM), in particular, has proved to be very convenient in order to extract relevant physical information such as the phase structure, order parameters, magnetic susceptibilities, correlation functions and critical exponents, among others [1, 2, 3, 4, 5].

In a recent paper [6], the effect of disorder has been investigated in the renormalized classical, Néel ordered, phase of the NLSM. This was done through the introduction of a random continuum distribution for the spin stiffness, which is the relevant control parameter in this phase. Quenched averages were then used to evaluate the effects of disorder on the correlation functions of quantum topological excitations (skyrmions). An interesting consequence was found concerning the energy gap of these excitations. In the Néel phase, without disorder, skyrmions have a finite energy gap, proportional to the spin stiffness $\left(\rho_{s}\right)$, which is the square of the staggered magnetization [7]. This fact usually enables one to use the skyrmion energy gap as a reliable order parameter for the Néel phase. When disorder is introduced, in such a way that magnetic dilution is not exponentially supressed, however, one finds that the skyrmion energy gap vanishes, in spite of the fact that the quenched staggered magnetization is different from zero [6]. This means that in certain situations one cannot use the skyrmion gap as a suitable order parameter for the AF order.

In the present work, we analyze the effects of disorder on the spin correlation function in the quantum paramagnetic (quantum disordered) phase of the NLSM and also on the staggered magnetic susceptibiliy, both in the Néel and quantum paramagnetic phases. For this purpose, we exploit the order-disorder duality relation existing between the spin excitations and the quantum skyrmion topological excitations [7]. Indeed, in the ordered Néel phase, skyrmions possess a nonzero energy gap while spinwaves (magnons) are gapless whereas in the quantum paramagnetic phase the former 
are gapless and the latter acquire a nonzero gap. The relevant control parameter of the system in the quantum paramagnetic phase is the spin gap at zero temperature, $\Delta_{s}$. Consequently, in order to describe the presence of disorder in the system in the quantum paramagnetic phase, we shall introduce a random distribution for the spin gap and evaluate quenched averages of relevant quantities. A further justification for this way of introducing disorder in the quantum paramagnetic phase comes from the way $\rho_{s}$ and $\Delta_{s}$ are expressed in terms of the coupling constant of the $\operatorname{NLSM}\left(g_{0}\right)$. Indeed, we have

$$
\rho_{s}=\frac{1}{g_{0}}-\frac{1}{g_{c}} ; \Delta_{s}=8 \pi\left(\frac{1}{g_{c}}-\frac{1}{g_{0}}\right),
$$

where $g_{c}$ is the critical coupling for the quantum phase transition separating the Néel and the quantum paramagnetic phases [2]. $g_{0}$ is directly related to the exchange coupling of the original spin system and therefore it is natural to expect that the effects of disorder existing in the latter should manifest through $g_{0}$ in the continuum version. Consequently, in view of (1), corresponding to a random distribution of $\rho_{s}$ in the Néel phase, we must have a similar distribution of $\Delta_{s}$ in the quantum paramagnetic phase. We shall use, both for the spin stiffness and spin gap, respectively in each phase, the distribution [6]

$$
P[\alpha]= \begin{cases}\frac{1}{N} \alpha^{\nu-1} e^{-\frac{\left(\alpha-\alpha_{s}\right)^{2}}{2 \sigma^{2}}} & \alpha \geq 0 \\ 0 & \alpha<0\end{cases}
$$

where $\nu>0$ and

$$
N=\sigma^{\nu} \Gamma(\nu) D_{-\nu}\left(-\frac{\alpha_{s}}{\sigma}\right) e^{-\frac{\alpha_{s}^{2}}{4 \sigma^{2}}}
$$

where $D_{-\nu}(x)$ is a parabolic cylinder function. In (2), $\alpha$ stands either for the spin stiffness $\rho$, in the ordered Néel phase $\left(\alpha_{s} \equiv \rho_{s}\right)$, or for the spin gap $\Delta$, in the quantum paramagnetic phase $\left(\alpha_{s} \equiv \Delta_{s}\right)$. Notice that the distribution vanishes for negative values of the argument. This is a natural choice since the control parameters $\rho$ and $\Delta$ are not defined in this case. Ferromagnetic couplings, in particular, are not described by the distribution above.

In the case of the high-Tc cuprates, which are prototypes of 2D quantum antiferromagnets, typical values for the parameters are [8, 9]: $\rho_{s} \simeq 10^{-1} \mathrm{eV} ; \Delta_{s} \simeq 10^{-3} \mathrm{eV}$. We 
assume $\sigma<<\rho_{s}$, and $\sigma<<\Delta_{s}$, respectively, in each phase, in order to ensure that the $\rho$ and $\Delta$ configurations are slowly varying and the continuum limit can be safely taken. Hence, typical values for the variance would be $\sigma \simeq 10^{-3} \mathrm{eV}$ and $\sigma \simeq 10^{-5} \mathrm{eV}$, respectively, in the ordered Néel and quantum paramagnetic phases. Also, for a sample of dimension $L \simeq 1 \mathrm{~mm}$ we have, in both phases, the relation (to be used later on)

$$
\left(\frac{L}{\hbar c}\right) \sigma>>\left(\frac{\alpha_{s}}{\sigma}\right)>>1,
$$

where $c$ is the spin-wave velocity, the characteristic velocity of the system. The typical value for the ordered phase is $\hbar c \simeq 1 \mathrm{eV} \AA[8]$ and we assume $\hbar c \simeq 10^{-2} \mathrm{eV} \AA$ in the quantum paramagnetic phase.

\section{2) Renormalized Classical Region}

Let us investigate here the effects of disorder on the homogeneous, static staggered susceptibility for $g_{0}<g_{c}$, or $\rho_{s}>0$. Within the $\mathrm{CP}^{1}$ formulation of the NLSM, this is given by [5]

$$
\chi(T)=\lim _{|\vec{k}|, \omega \rightarrow 0} \chi(\omega, \vec{k}, T)=\frac{T}{4 m^{2}(T)},
$$

where $\chi(\omega, \vec{k}, T)$ is the vacuum polarization scalar for the $\mathrm{CP}^{1}$ constraint field and $m(T)$ is the spin gap. For $T<<\rho_{s}$, we have $m(T)=T e^{-\frac{2 \pi \rho_{s}}{T}}$ and

$$
\chi(T)=\frac{e^{\frac{4 \pi \rho_{s}}{T}}}{4 T} \stackrel{T \rightarrow 0}{\longrightarrow} \infty
$$

The spin gap vanishes at $T=0$ and the susceptibility diverges, implying the occurence of an ordered Néel state at zero temperature.

In the presence of disorder, the quenched susceptibility will be given by

$$
\bar{\chi}(T)=\int_{0}^{\infty} d \rho P[\rho] \chi(\rho, T),
$$

where $P[\rho]$ is given by (2). The condition $\sigma<<\rho_{s}$ enables us to use the expression (6) in (7), obtaining, for $T<<\sigma<<\rho_{s}$

$$
\bar{\chi}(T)=\frac{1}{4 T^{\nu}}\left[\frac{4 \pi \sigma^{2}}{\rho_{s}}+T\right]^{\nu-1} \exp \left[\frac{8 \pi^{2} \sigma^{2}}{T^{2}}+\frac{4 \pi \rho_{s}}{T}\right],
$$


We see that we have $\bar{\chi}(T) \stackrel{T \rightarrow 0}{\longrightarrow} \infty$. This means that even after the inclusion of disorder the ordered Néel state persists for $\rho_{s}>0$, a result that confirms, from the point of view of the susceptibility, the observation made in [6] by looking directly at the quenched magnetization. It has been shown, nevertheless, that the quantum skyrmion quenched correlation function changes its behavior from exponential to power-law decay at large distances as a consequence of the presence of disorder in this phase [6].

\section{3) Quantum Paramagnetic Phase}

We now consider the effects of disorder on the susceptibility in the quantum paramagnetic phase, characterized by $g_{0}>g_{c}$, or $\Delta_{s}>0 . \chi(T)$ is still given by (5) but the spin gap is now given, for $T<<\Delta_{s}$, by [4]

$$
m(T)=\Delta_{s}+2 T e^{-\frac{\Delta_{s}}{T}}
$$

and no longer vanishes at zero temperature. The susceptibility is now given by

$$
\chi(T)=\frac{T}{4 \Delta_{s}^{2}(T)}\left(1-4 \frac{T}{\Delta_{s}} e^{-\frac{\Delta_{s}}{T}}\right)
$$

and we see that $\chi(T) \stackrel{T \rightarrow 0}{\longrightarrow} 0$, implying the absence of an ordered state at $T=0$ in this phase.

We now introduce disorder in the system and consider the quenched susceptibility

$$
\bar{\chi}(T)=\int_{0}^{\infty} d \Delta P[\Delta] \chi(\Delta, T)
$$

where $P[\Delta]$, the random distribution of $\Delta$ 's, is given by $(2)$ and $\chi(\Delta, T)$, by $(10)$. Observe that the condition $\sigma<<\Delta_{s}$ allows us to introduce the $T / \Delta_{s}$-expanded expression (10) inside the integral in (11). After performing the $\Delta$ integration we get, for $T<<\Delta_{s}$

$$
\bar{\chi}(T)=\frac{T}{4 \Delta_{s}^{2}}-\frac{T^{2}}{\Delta_{s}^{3}} \exp \left[-\frac{\Delta_{s}}{T}\left(1-\frac{\sigma^{2}}{2 T \Delta_{s}}\right)\right]\left[1-(\nu-4) \frac{\sigma^{2}}{T \Delta_{s}}\right] .
$$

We see that $\bar{\chi}(T) \stackrel{T \rightarrow 0}{\longrightarrow} 0$, implying that the inclusion of disorder, described by (2) does not change the fact that the system is in a disordered ground state, at $T=0$, for 
$\Delta_{s}>0$ as one should expect. Notice, however, that the subleading behavior of the susceptibility is modified by the presence of disorder. Observe, on the other hand, that disorder does not change the critical exponent for the quantum phase transition occurring for $g \rightarrow g_{c}$, at $T \rightarrow 0$. In any case, we have the susceptibility behaving as $\left(g-g_{c}\right)^{-2}$ at the critical point.

Let us investigate now the large distance behavior of the spin correlation function $<S_{z}(\vec{x}, \tau) S_{z}(\overrightarrow{0}, \tau)>$. This is proportional to [5]

$$
\chi(\vec{x})=\int \frac{d^{2} k}{(2 \pi)^{2}} \chi(\omega=0, \vec{k}, T) e^{i \vec{k} \cdot \vec{x}|\vec{x}| \rightarrow \infty} \longrightarrow e^{-2 m(T)|\vec{x}|}
$$

where $m(T)$ is given by (9) in the quantum paramagnetic phase.

We are interested in the zero temperature, large distance behavior of the quenched spin correlation function, hence we consider

$$
\bar{\chi}(\vec{x})=\int_{0}^{\infty} d \Delta P[\Delta] \chi(\vec{x}, \Delta, T=0) \stackrel{|\vec{x}| \rightarrow \infty}{\longrightarrow} \int_{0}^{\infty} d \Delta P[\Delta] e^{-2 \Delta|\vec{x}|} .
$$

Performing the $\Delta$ integration, we get, for $\sigma<<\Delta_{s}$,

$$
\bar{\chi}(\vec{x})=\frac{\Gamma(\nu)}{\sqrt{2 \pi}}\left(\frac{\sigma}{\Delta_{s}}\right)^{\nu-1} e^{-\Delta_{s}^{2} / 2 \sigma^{2}} \exp \left[\left(\sigma|\vec{x}|-\frac{\Delta_{s}}{2 \sigma}\right)^{2}\right] D_{-\nu}\left(2 \sigma|\vec{x}|-\frac{\Delta_{s}}{\sigma}\right) .
$$

In order to obtain the large distance behavior of (15), we use condition (4) and the asymptotic behavior of the parabolic cylinder functions [10]. The result is

$$
\bar{\chi}(\vec{x})=\stackrel{|\vec{x}| \rightarrow \infty}{\longrightarrow} \frac{\Gamma(\nu)}{2^{\nu} \sqrt{2 \pi}}\left(\frac{\Delta_{s}^{1-\nu}}{\sigma}\right) e^{-\Delta_{s}^{2} / 2 \sigma^{2}} \frac{1}{|\vec{x}|^{\nu}}\left[1+\nu \frac{\Delta_{s}}{2 \sigma^{2}|\vec{x}|}\right]
$$

The power-law large distance behavior of the quenched spin correlation function indicates that, in the presence of disorder, associated to the distribution of $\Delta$ 's given by (2), the spin excitations become gapless for $\Delta_{s}>0$ and $T=0$. This happens, in spite of the fact that the system is in a disordered state, as we have seen before. In such sates, the spin excitations usually have a gap and therefore, the introduction of disorder completely changes the scenario in this case. As we have mentioned something similar happened with the skyrmion topological excitations in the renormalized classical region thereby clearly exposing the duality existing between skyrmions and spin excitations. 


\section{4) Concluding Remarks}

We have considered the inclusion of disorder in a two-dimensional quantum antiferromagnet, both in the renormalized classical and quantum paramagnetic phases, at low temperatures. Staggered magnetic susceptibilities and spin correlation functions have been studied in the quenched regime. The behavior of these indicate that the nature of the ground state is not modified but, nevertheless, the properties of the basic excitations are deeply changed. Spin excitations, become gapless in the quantum paramagnetic phase, thereby exhibiting a dual character with respect to skyrmion quantum excitations, which become gapless, in the presence of disorder, within the renormalized classical region [6].

In this work, we described the presence of disorder by means of a continuuum random distribution of the relevant control parameter in each phase, either the spin stiffness or the zero-temperature-spin-gap. It would be interesting to investigate the effect of a discrete distribution thereof, which could be introduced by delta functions. Also, the types of disorder in which ferromagnetic couplings would be allowed would be worth studying and could have interesting consequences for models of spin-glasses. We are presently considering these possibilties.

\section{Acknowledgements}

This work has been supported in part by CNPq, FAPERJ and PRONEX-66.2002/19989. CMSC has been supported by CAPES and FAPERJ and ECM has been partially supported by CNPq.

\section{References}

[1] F.D.M.Haldane, Phys. Rev. Lett. 50, 1153 (1983); F.D.M.Haldane, Phys. Lett., A93 464 (1983)

[2] S.Chakravarty, B.Halperin and D.Nelson, Phys. Rev. B39, 2344 (1989) 
[3] N.Read and S.Sachdev, Phys. Rev. B42, 4568 (1990); A.V.Chubukov, S.Sachdev and J.Ye, Phys. Rev. B49, 11919 (1994); V.Y.Irkhin and A.A.Katanin, Phys. Rev. B55, 12318 (1997)

[4] A.V.Chubukov and O.Starykh, Phys. Rev. B52, 440 (1995)

[5] O.Starykh, Phys. Rev. B50, 16428 (1994)

[6] E.C.Marino, Phys. Rev. B65, 054418 (2002)

[7] E.C.Marino, Phys. Rev. B61, 1588 (2000)

[8] A.P.Kampf, Phys. Rep. 249, 219 (1994)

[9] E.C.Marino and M.B.Silva Neto, Phys. Rev. B66, 224512 (2002)

[10] I.S.Gradshteyn and I.M.Ryzhik, "Table of Integrals, Series and Products", Academic Press, New York, 1980 